\title{
Caracterización y frecuencia de cataratas en perros en Medellín, Colombia (2019-2020)
}

\author{
Characterization and frequency of cataracts in dogs in Medellín, Colombia
} (2019-2020)

\author{
Johanna Correa ${ }^{1,5}$, Laura Carolina Álvarez ${ }^{2}$, Sandra Patricia Acevedo ${ }^{3,4}$, \\ Nathalia María Correa-Valencia ${ }^{4}$
}

\section{RESUMEN}

El objetivo del presente estudio fue reportar la frecuencia y caracterización de cataratas diagnosticadas en perros que asistieron a consulta a la Unidad Oftalmológica Ocularvet, en la ciudad de Medellín (Antioquia, Colombia) entre agosto de 2019 y agosto de 2020. Se realizó un muestreo a conveniencia, con la inclusión de perros de todas las edades con diagnóstico de catarata y la exclusión de aquellos con tratamientos previos a la misma o con patologías que dificultaran la evaluación del cristalino. Se caracterizó demográficamente la población (raza, sexo, edad, enfermedades previas/concomitantes), además de la información relacionada con la catarata (ojo afectado, ubicación de la catarata, grado de desarrollo). Se realizó una evaluación oftalmológica a 442 perros durante el año de estudio, de los cuales el 36\% fue diagnosticado con cataratas, representando 203 ojos afectados. Perros machos $(54.1 \%)$, de raza Poodle (11.9\%), Schnauzer $(10.1 \%)$ y mestizos $(8.2 \%)$, fueron los principalmente afectados. La frecuencia de casos entre los

${ }^{1}$ Grupo de Investigación Veterinaria Remington GINVER, Facultad de Medicina Veterinaria, UniRemington, Medellín, Colombia

${ }^{2}$ Facultad de Ciencias Administrativas y Agropecuarias, Corporación Universitaria Lasallista, Caldas, Colombia

${ }^{3}$ Unidad Oftalmológica Ocularvet, Medellín, Colombia

${ }^{4}$ Grupo Centauro, Escuela de Medicina Veterinaria, Facultad de Ciencias Agrarias, Universidad de Antioquia, Medellin, Colombia

${ }^{5}$ E-mail: johanacorporativo@gmail.com

Recibido: 3 de marzo de 2021

Aceptado para publicación: 5 de septiembre de 2021

Publicado: 27 de octubre de 2021

CLos autores. Este artículo es publicado por la Rev Inv Vet Perú de la Facultad de Medicina Veterinaria, Universidad Nacional Mayor de San Marcos. Este es un artículo de acceso abierto, distribuido bajo los términos de la licencia Creative Commons Atribución 4.0 Internacional (CC BY 4.0) [https:// creativecommons.org/licenses/by/4.0/deed.es] que permite el uso, distribución y reproducción en cualquier medio, siempre que la obra original sea debidamente citada de su fuente original 
grupos etarios antes de los 3 años de edad fue similar y se incrementó a partir de los 7 años. Diez de los 159 pacientes $(6.3 \%)$ presentaron enfermedades previas o concomitantes al momento del diagnóstico de catarata, siendo la más frecuente la diabetes mellitus tipo I (2.5\%). La ubicación nuclear (23.2\%) fue la más frecuente. Así mismo, el grado de desarrollo incipiente (44.3\%) y la catarata madura $(30.5 \%)$ fueron las de mayor frecuencia. Se concluye que el grado de desarrollo incipiente fue la forma más común de catarata, sin una distribución definida por raza, sexo o edad.

Palabras clave: bienestar animal, cristalino, enfermedad ocular, pérdida visual

\section{AbSTRACT}

The aim of this study was to report the frequency and characterization of cataracts diagnosed in dogs that attended consultation at the Ocularvet Ophthalmological Unit, in the city of Medellín (Antioquia, Colombia) between August 2019 and August 2020. A sampling was carried out at convenience, with the inclusion of dogs of all ages with a diagnosis of cataract and the exclusion of those with previous treatments or with pathologies that make it difficult to evaluate the lens. The population was demographically characterized (breed, sex, age, previous / concomitant diseases), in addition to information related to cataract (affected eye, cataract location, degree of development). An ophthalmological evaluation was performed on 442 dogs during the study year, of which $36 \%$ were diagnosed with cataracts, representing 203 affected eyes. Male dogs (54.1\%), Poodle (11.9\%), Schnauzer (10.1\%) and crossbreds $(8.2 \%)$ were mainly affected. The frequency of cases between the age groups before 3 years of age was similar and increased after 7 years. Ten of the 159 patients $(6.3 \%)$ had previous or concomitant diseases at the time of cataract diagnosis, the most frequent being type I diabetes mellitus (2.5\%). The nuclear location $(23.2 \%)$ was the most frequent. Likewise, the degree of incipient development (44.3\%) and mature cataract (30.5\%) were the most frequent. It is concluded that the degree of incipient development was the most common form of cataract, without a defined distribution by breed, sex or age.

Key words: animal welfare, crystalline, eye disease, visual loss

\section{INTRODUCCIÓN}

La importancia que han adquirido los perros como animales de compañía y su estrecho vínculo con los seres humanos ha generado un mayor interés por parte de los propietarios sobre el cuidado, manejo y salud de sus mascotas, lo que resalta la necesidad de avanzar en el conocimiento médico y en técnicas asertivas de abordaje diagnóstico y terapéutico para las patologías que los afectan (Gómez et al., 2007). Entre estas, surge la necesidad de innovar especialidades médicas como la oftalmología veterinaria para la creación de tratamientos médicos y quirúrgicos que mejoren la calidad de vida de los pacientes con enfermedades oculares (Bayón del Río et al., 2010).

Entre las estructuras oculares de mayor interés se encuentra el cristalino, una estructura biconvexa, transparente y avascular ubicada en el segmento anterior del ojo (Mellersh, 2014). El cristalino es responsable de la refracción de la luz, haciendo que esta se enfoque en la retina, cumpliendo así con la función de lente ocular natural, fundamental 
para la visión de los animales (Gelatt, 2003; Mellersh, 2014). Por lo tanto, su transparencia es una propiedad crucial para que cumpla su función de manera adecuada (Mellersh, 2014).

Las cataratas se encuentran entre las enfermedades oculares más comunes y son la principal causa de pérdida de visión en humanos y perros (Gelatt, 2003; Espejo y Esteban, 2008; Guerra et al., 2018). Se denomina catarata a la pérdida de transparencia del cristalino, lo que da lugar a la presencia de opacidades en uno o ambos ojos (Gelatt y MacKay, 2005; Herrera, 2007; Raghuvanshi y Maiti, 2013). El cambio de color aparente del cristalino (leucocoria) es uno de los motivos de consulta más frecuente en la medicina de pequeñas especies (Espejo y Esteban, 2008). La degeneración del cristalino es un proceso que se presenta por disturbios metabólicos, ocurre un aumento de las enzimas proteolíticas que causan daño en las membranas celulares y la degradación de las proteínas del lente (Gomes et al., 2017). Las cataratas pueden desarrollarse por múltiples causas, incluyendo senilidad, trauma, inflamación, secuela de diabetes, uso constante de esteroides y causas genéticas (Glover y Constantinescu, 1997; Gelatt y MacKay, 2005; Davidson y Nelms, 2013).

Los perros que sufren esta alteración tienden a mantenerse cerca a los propietarios y presentan una aproximación mucho más cuidadosa al ambiente que lo rodea (Gelatt, 2003). Adicionalmente, patrones como la desorientación, tropiezos, golpes frecuentes contra objetos y la no atención de pautas de juego se presentan con mayor frecuencia. Cuando esto sucede y el propietario lo detecta, el animal ya ha perdido entre el 40 y $50 \%$ de la funcionalidad visual (Del Sole, 2014).

Dada la evolución de la oftalmología veterinaria en las últimas décadas, se ha logrado abordar esta dolencia y ampliar el conocimiento sobre su fisiopatología. Es por esto que el presente estudio tuvo como objetivo reportar la frecuencia y caracterización de cataratas diagnosticadas en perros que asistieron a consulta a la Unidad Oftalmológica Ocularvet de la ciudad de Medellín (Antioquia, Colombia).

\section{Materiales y Métodos}

\section{Consideraciones Éticas}

De acuerdo con el Art. 11 de la Resolución N. 8430 de 1993 de la República de Colombia, este proyecto de investigación se considera sin riesgo, ya que utiliza los resultados obtenidos a partir de historias clínicas. No se tuvo contacto ni se requirieron nuevas intervenciones con los animales. No se colectó información personal de los propietarios.

\section{Tipo de Estudio}

Se realizó un estudio descriptivo retrospectivo a partir de un muestreo a conveniencia de los pacientes diagnosticados con cataratas que se presentaron al servicio médico de la Unidad oftalmológica Ocularvet (Medellín, Colombia), entre agosto de 2019 y agosto de 2020. Los perros debían contar con un diagnóstico clínico de catarata, independiente de la edad, sexo, raza y tipo de catarata. Los individuos con tratamientos previos para la patología de interés, pacientes con patologías asociadas que no permitían la observación del cristalino (e.g. glaucoma, sinequias, uveítis facolítica, membrana fibrovascular) y pacientes diagnosticados con patologías oculares diferentes a catarata (e.g. queratoconjuntivitis seca, glaucoma, uveítis, queratitis pigmentaria, prolapso de tercer párpado) fueron excluidos del estudio.

\section{Definición de Caso: Diagnóstico de Ca- tarata}

Como parte de la consulta oftalmológica de rutina, el paciente pasa por una evaluación del sistema ocular, incluyendo la evalua- 
ción del reflejo pupilar directo y consensual, evaluación de reflejos monocromáticos (azul y rojo), evaluación de la respuesta de amenaza y sorteo de obstáculos (a necesidad), prueba de Schirmer (para medir producción lagrimal) y tonometría de aplanación o Goldmann (para verificar la presión intraocular). Una vez realizadas estas pruebas se procede a la aplicación de una gota de Alcaine $^{\circledR}$ (proparacaína) para evaluar la presión intraocular, y Midriacyl ${ }^{\circledR}$ (tropicamida) para dilatar la pupila y poder observar el fondo de ojo, especialmente cuando las cataratas abarcaban áreas periféricas corticales o cercanas al ecuador del globo ocular. Adicionalmente, se realiza evaluación de la córnea, párpados y anexos oculares con transiluminador y lupa.

Una vez dilatada la pupila se procede a realizar una oftalmoscopia a luz penumbra y se evalúa la cámara anterior y posterior, el cristalino y el fondo de ojo. En caso de considerarse necesario por parte del especialista, se realizaba una evaluación más exhaustiva del fondo del ojo, mediante retinoscopio y panoftalmoscopio. Por último, en caso de ser necesario, se aplica tinción con fluoresceína a nivel de la córnea para la detección de rupturas en la membrana celular epitelial y en el contacto célula-célula, o Rosa de Bengala para la evaluación de las células epiteliales, cuando la capa protectora de mucina ha desaparecido o es anormal (Nelson, 2004).

Se realiza ultrasonografía ocular en aquellos pacientes diagnosticados con cataratas, descartando desprendimiento vítreo, coroideo o de retina, evaluando la condición vítrea, así como la posición del cristalino, y la medición del saco capsular para determinar tamaño del lente intraocular a instalar (en caso de ser considerado). Adicionalmente, se realiza electrorretinografía bajo sedación, evaluando la actividad eléctrica de la retina mediante la medición de la onda A (condición de conos y bastones) y onda B (células de la glía), confirmando su funcionalidad previa al tratamiento quirúrgico. Por último, se realiza una gonioscopia para evaluar el ángulo iridiocorneal y prever posibles complicaciones asociadas al glaucoma.

Según el grado de desarrollo, las cataratas se clasifican como incipiente (opacidad focal pequeña), inmadura (opacidad de mayor extensión), madura (opacidad que rodea todo el cristalino) o hipermadura (presenta proteólisis y reabsorción de la lente) (Gelatt, 2003).

\section{Análisis Estadístico}

Se colectaron datos demográficos de la población (i.e. raza, sexo, edad, enfermedades previas/concomitantes), además de la información relacionada con la caracterización de la catarata (i.e. ojo afectado, ubicación de la catarata, grado de desarrollo) a partir de las historias clínicas colectadas durante el tiempo de observación. Los datos fueron registrados manualmente en hojas de cálculo de MS Excel y luego se exportaron a Stata 16.0 (StataCorp 2020, USA) para el análisis estadístico descriptivo.

\section{Resultados}

A la consulta asistieron 442 perros para evaluación oftalmológica durante el año de estudio. De estos, 159 (36\%) fueron diagnosticados con catarata, logrando un total de 203 ojos afectados. Los grupos raciales con mayor frecuencia de cataratas fueron el Poodle (19/159), Schnauzer (16/159) y el mestizo (13/159), principalmente machos $(86 / 159 ; 54.1 \%)$, con una distribución aparentemente uniforme entre los grupos etarios menores o iguales a tres años, pero con un incremento en la presentación de cataratas a partir de los 7 años de edad (Cuadro 1). Diez de los 159 pacientes presentaban enfermedades previas o concomitantes al momento del diagnóstico de catarata, siendo la más frecuente la diabetes mellitus tipo I (4/159), seguido de trauma ocular (uveitis, úlcera corneal) y hemoparasitosis (anaplasmosis, ehrlichiosis). 
Cuadro 1. Población de perros con diagnóstico de catarata en la ciudad de Medellín, Colombia, según grupo racial, sexo y edad (2019-2020)

\begin{tabular}{|c|c|c|c|}
\hline Variable & Grupos raciales & $\begin{array}{c}\text { Casos } \\
\text { (n) }\end{array}$ & $\begin{array}{c}\text { Porcentaje } \\
(\%)\end{array}$ \\
\hline \multirow[t]{16}{*}{ Raza } & Poodle & 19 & 11.9 \\
\hline & Schnauzer & 16 & 10.1 \\
\hline & Mestizo & 13 & 8.2 \\
\hline & Bulldog Francés & 8 & 5 \\
\hline & Shih-Tzu & 8 & 5 \\
\hline & Boston Terrier & 8 & 5 \\
\hline & Pinscher & 6 & 3.8 \\
\hline & Cocker Spaniel & 5 & 3.1 \\
\hline & Golden Retriever & 5 & 3.1 \\
\hline & Labrador Retriever & 5 & 3.1 \\
\hline & Husky Siberiano & 4 & 2.5 \\
\hline & Beagle & 3 & 1.9 \\
\hline & Bulldog Inglés & 3 & 1.9 \\
\hline & Otras & 20 & 12.6 \\
\hline & No reporta & 36 & 22.6 \\
\hline & Total & 159 & 100 \\
\hline \multirow[t]{3}{*}{ Sexo } & Macho & 86 & 54.1 \\
\hline & Hembra & 73 & 45.9 \\
\hline & Total & 159 & 100 \\
\hline \multirow{13}{*}{$\begin{array}{l}\text { Edad } \\
\text { (en años) }\end{array}$} & $<1$ & 1 & 0.6 \\
\hline & $\geq 1-\leq 2$ & 4 & 2.5 \\
\hline & $>2-\leq 3$ & 7 & 4.4 \\
\hline & $>3-\leq 4$ & 8 & 5 \\
\hline & $>4-\leq 5$ & 5 & 3.2 \\
\hline & $>5-\leq 6$ & 8 & 5 \\
\hline & $>6-\leq 7$ & 8 & 5 \\
\hline & $>7-\leq 8$ & 16 & 10.1 \\
\hline & $>8-\leq 9$ & 10 & 6.3 \\
\hline & $>9-\leq 10$ & 22 & 13.8 \\
\hline & $>10$ & 66 & 41.6 \\
\hline & No reporta & 4 & 2.5 \\
\hline & Total & 159 & 100 \\
\hline
\end{tabular}

Se encontró que la presentación unilateral (tanto del ojo izquierdo como del derecho) fue más frecuente que la presentación bilateral. La ubicación más frecuente de la catarata fue la nuclear $(47 / 203 ; 23.2 \%)$, mientras que el grado de desarrollo incipiente $(90 / 159 ; 44.3 \%)$ y la catarata madura $(62 /$ $159 ; 30.5 \%)$ representaron las mayores proporciones (Cuadro 2). 
Cuadro 2. Población de perros con diagnóstico de catarata en la ciudad de Medellín, Colombia, según las características de la patología presente (2019-2020)

\begin{tabular}{llcc}
\hline \multirow{2}{*}{ Variable } & & $\begin{array}{c}\text { Casos } \\
(\mathrm{n})\end{array}$ & $\begin{array}{c}\text { Porcentaje } \\
(\%)\end{array}$ \\
\hline \multirow{2}{*}{ Ojo afectado } & Unilateral - ojo derecho & 68 & 33.5 \\
& Unilateral - ojo izquierdo & 68 & 33.5 \\
& Bilateral & 67 & 33 \\
\cline { 2 - 4 } & Total & 203 & 100 \\
\hline \multirow{2}{*}{ Ubicación de la } & Nuclear & 47 & 23.2 \\
& Capsular & 31 & 15.3 \\
& Cortical & 21 & 10.3 \\
& Capsular posterior & 13 & 6.4 \\
& Nuclear tumefacta & 7 & 3.4 \\
& Polar & 2 & 1 \\
& Polar posterior & 2 & 1 \\
& No reporta & 80 & 39.4 \\
\cline { 2 - 4 } & Total & 203 & 100 \\
\hline \multirow{2}{*}{ Grado de desarrollo la catarata } & Incipiente & 90 & 44.3 \\
& Madura & 62 & 30.5 \\
& Incipiente tardía & 30 & 14.8 \\
& Hipermadura & 18 & 8.9 \\
& Inmadura & 2 & 1 \\
& Inmadura tardía & 1 & 0.5 \\
\cline { 2 - 4 } & Total & 203 & 100 \\
\hline
\end{tabular}

Las Figuras que se presentan a continuación corresponden a los casos observados en la población de interés durante el periodo de estudio. La Figura 1 presenta fotografías de las ubicaciones y grados de desarrollo de las cataratas diagnosticadas en la población de estudio. La Figura 2 muestra cataratas con grado de desarrollo incipiente, particularmente en perros entre 3 y 5 años con deterioro del enfoque, pero aún con capacidad visual conservada. La Figura 3 muestra cataratas con grado de desarrollo hipermaduro-reabsortivas, particularmente en perros entre los 7 y 10 años, con déficit visual completo. La Figura 4 muestra catara- tas con grado de desarrollo maduro de origen inflamatorio, particularmente en perros con enfermedades sistémicas asociadas a hemoparasitosis y diabetes mellitus tipo I, con déficit visual completo.

\section{Discusión}

El hallazgo de cataratas en el ejercicio práctico veterinario a veces suele ser incidental, ya que cuando se evidencia un déficit visual en el perro, el cristalino se encuentra afectado en más del 50\% (Bayón del Río et al., 2010). Por ello es importante que el mé- 

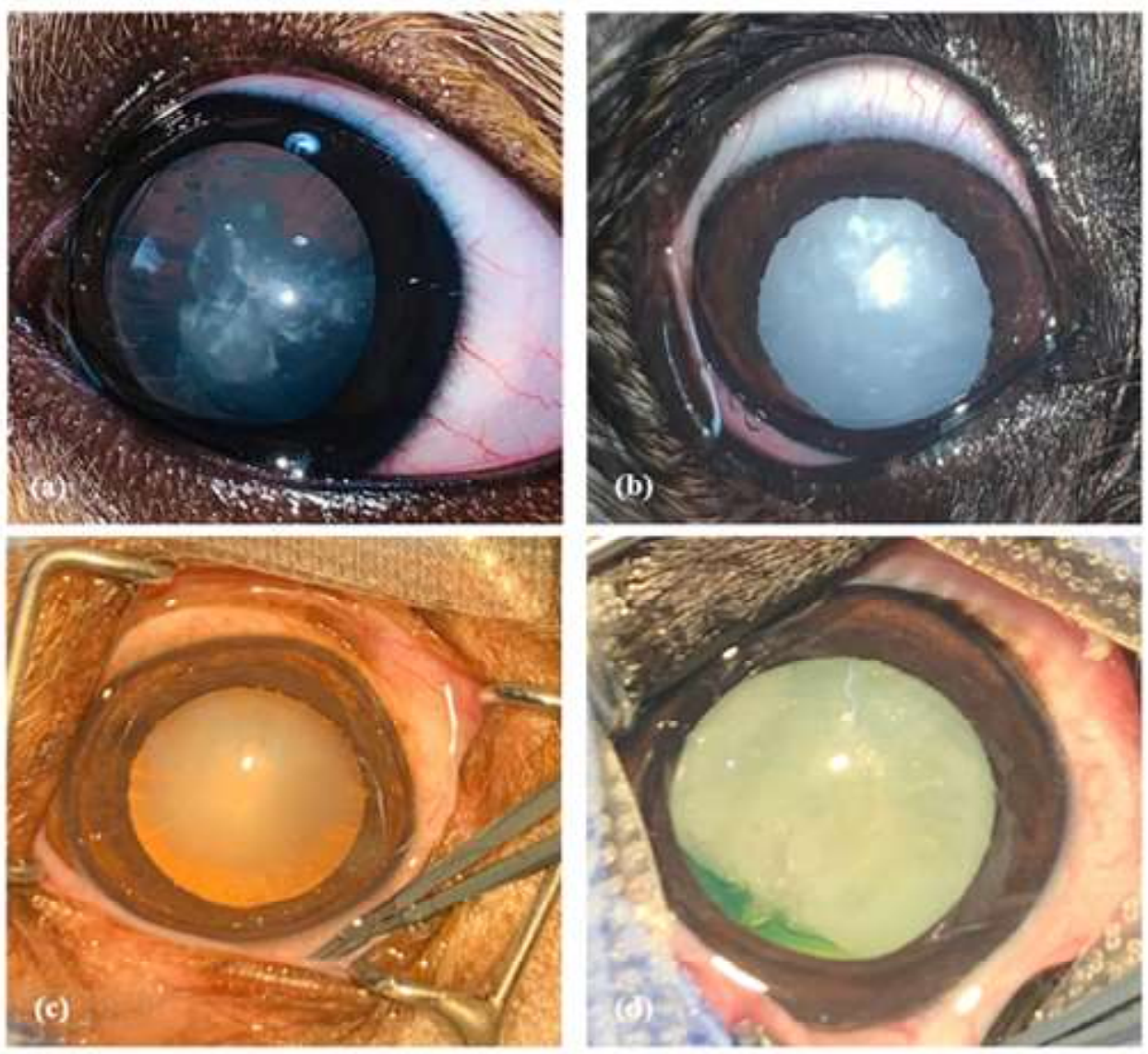

Figura 1. Grados de desarrollo de cataratas en perros, Medellín, Colombia: (a) catarata capsular incipiente que afecta la parte posterior del cristalino; (b) catarata nuclear madura evaluada con iluminación difusa directa; (c) catarata incipiente tardía nuclear; (d) catarata hipermadura reabsortiva (obsérvense la alteración en la superficie inferior del cristalino)

dico veterinario aborde todos los sistemas de manera integral y relacione patologías sistémicas con una posible afección ocular. Asimismo, si bien muchos perros son llevados a consulta por la leucocoria y la conducta errática, es importante recalcar que la opacidad del cristalino también puede encontrarse en pacientes con enfermedades metabólicas como la diabetes mellitus tipo I, procesos inflamatorios oculares y traumáticos, o con presencia de hemoparásitos como Erlichia spp y Hepatozoon canis (Komnenou et al., 2007; Acevedo et al., 2009) y de allí la importancia de no abordar la patología ocular únicamente de forma local.
Las cataratas pueden presentarse de forma unilateral o bilateral, siendo la presentación unilateral una de las causales de pérdida visual más comunes, tal y como se reporta en el presente estudio. Esto se debe a que el perro no presenta sintomatología asociada o cambio comportamental que alerte a los propietarios, dado que conserva su visión en uno de los ojos.

Reportes de la literatura científica señalan la ocurrencia de cataratas en diversas razas (Gelatt y MacKay, 2005; Donzel et al., 2017). En el presente estudio, las razas más afectadas fueron el Poodle y Schnauzer, así 

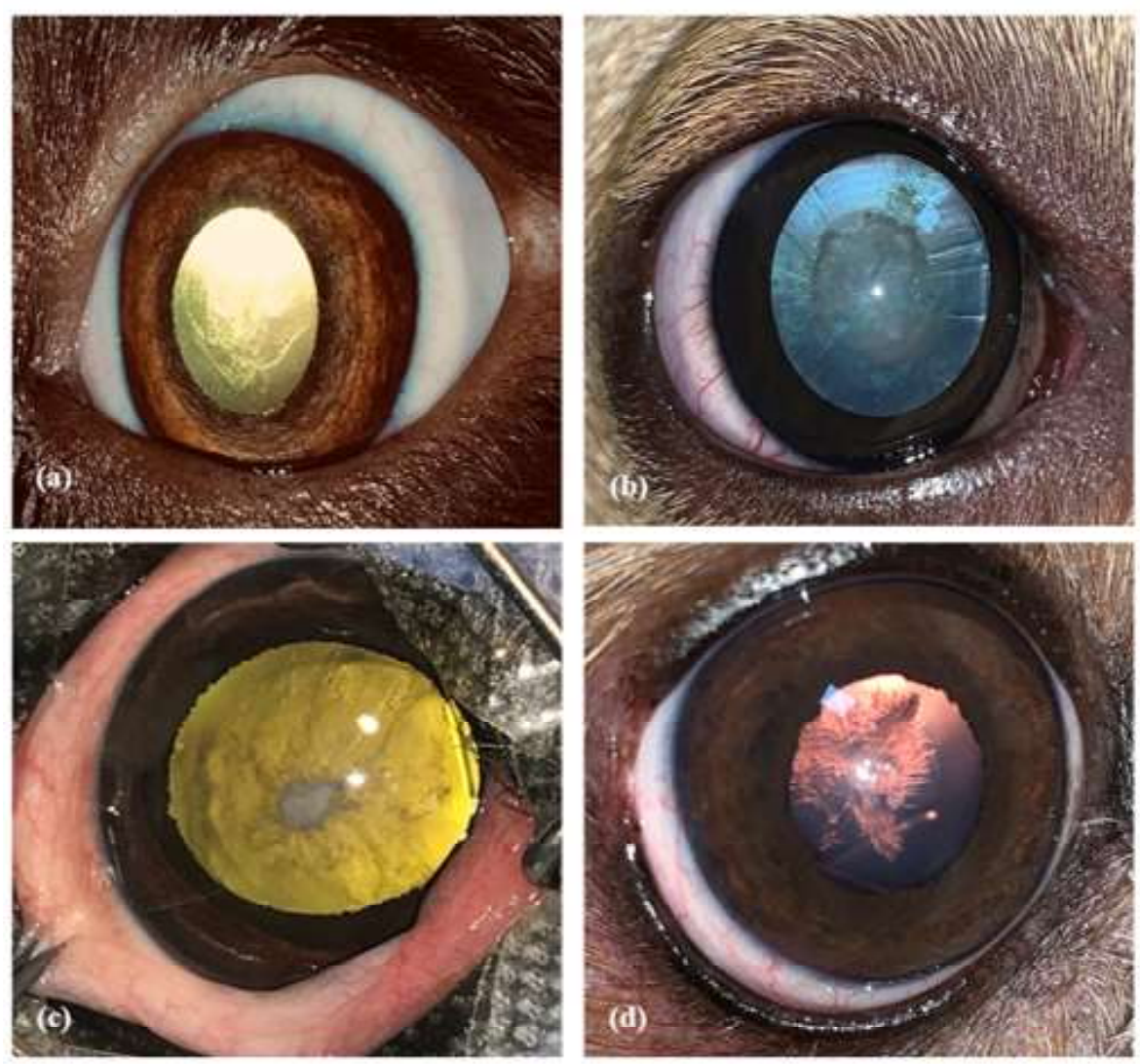

Figura 2. Cataratas con un grado de desarrollo incipiente en perros (Medellín, Colombia): (a) catarata capsular posterior (obsérvese las líneas que delimitan la cápsula; (b) catarata cortical en forma radial; (c) catarata capsular (la opacidad del cristalino se observa oscura); (d) catarata nuclear focal

como los perros mestizos, seguidos por pacientes de razas braquicefálicas (Bulldog Francés, Shih-Tzu, Boston Terrier), los cuales agrupan el $45.2 \%$ del total de perros evaluados, lo cual concuerda con la literatura (Donzel et al., 2017). Así mismo, se reporta que las razas puras están más predispuestas a esta patología (Silva, 2010; Donzel et al., 2017). Esta patología tiene un componente hereditario (Bayón del Río et al., 2010), de ahí la importancia de no reproducir ejemplares que presenten este cuadro.

Una clasificación de las cataratas es por la edad, teniéndose de esa manera las cataratas congénitas (desde el nacimiento), infantiles (0-1 año), juveniles (1-5 años) y las ca- taratas seniles (más de 5 años) (Glover et al., 1995; Gelatt K y Gelatt N, 2011; Davidson y Nelms, 2013). En el presente estudio se encontró un paciente con catarata infantil. La catarata juvenil representó el $20.1 \%$ del total de la muestra del presente estudio. De acuerdo con la experiencia de los autores, muchos de los pacientes no tienen como signo principal la leucocoria al momento de la consulta, sino el comportamiento errático al caminar, jugar o reconocer al propietario. Adicionalmente, el hallazgo de cataratas es generalmente incidental en perros jóvenes, ya que son llevados por problemas iniciales de córnea y epifora y la catarata se define como el diagnóstico definitivo tras un examen especializado del globo ocular. La catarata senil 

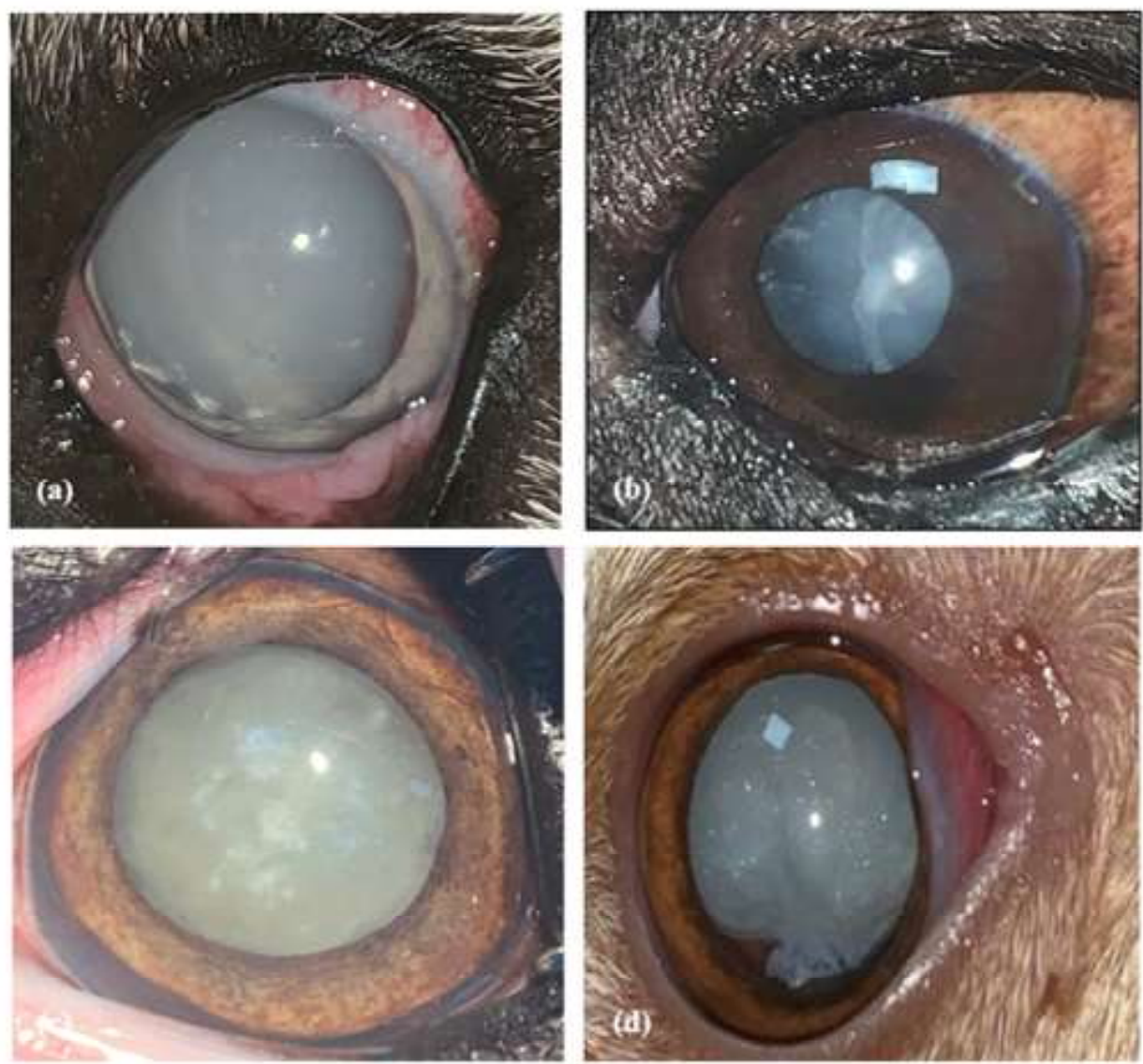

Figura 3. Cataratas con un grado de desarrollo hipermaduro en perros (Medellín, Colombia): (a) catarata clásica (obsérvese la rugosidad del cristalino); (b) catarata con alteración de la superficie capsular; (c) catarata con calcificación capsular; (d) catarata reabsortiva con ruptura capsular

se observó en la mayoría de los casos (76.7\%), correspondiendo con la frecuencia de consulta de pacientes seniles con leucocoria $y$ otras degeneraciones oftalmológicas

Es importante destacar que muchas de las cataratas son el resultado de enfermedades concomitantes como la diabetes o de procesos inflamatorios a nivel ocular, cuyo riesgo también aumenta con la edad (Abrams et al., 2011; Yoon et al., 2020). En el presente estudio, las enfermedades previas o concomitantes que pudieron predisponer a la pre- sentación de la catarata, la diabetes mellitus tipo I fue la más frecuente, seguido del trauma ocular y la hemoparasitosis. La diabetes mellitus tipo I lleva a la acumulación excesiva de glucosa en el cristalino generando alteración de proteínas cristalinas, lo cual favorece la aparición precoz y acelerada de las cataratas y, en el peor escenario, un daño a la retina (Lima y Ríos, 2004). Se estima que entre el 70 y $80 \%$ de los perros diabéticos desarrollarán cataratas en algún momento de su vida, usualmente de forma bilateral y de progresión aguda (Abrams et al., 2011). 

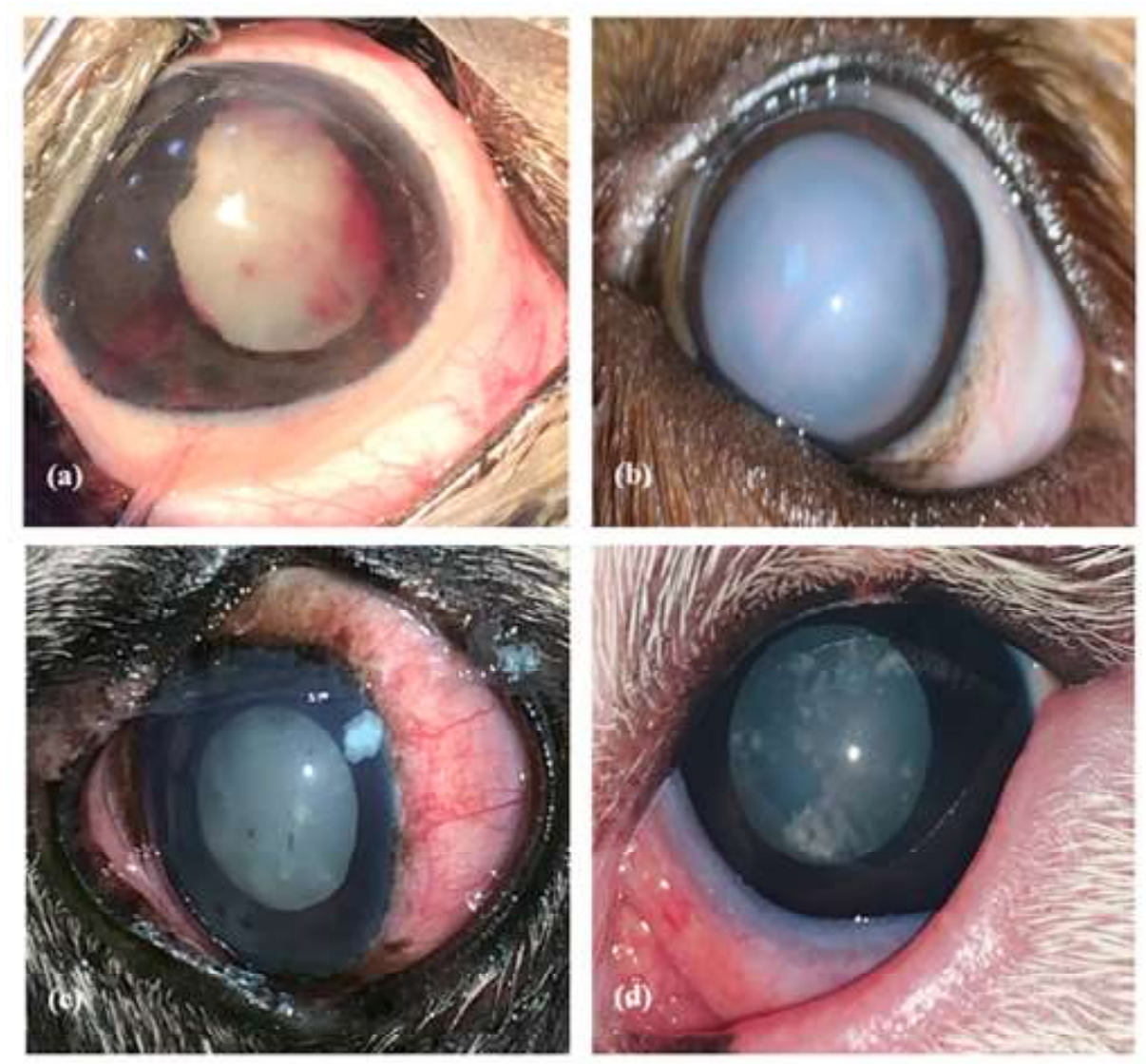

Figura 4. Cataratas maduras de origen inflamatorio en perros (Medellín, Colombia): (a) catarata en paciente diabético con uveítis facoclástica; (b) catarata secundaria a uveitis por Ehrlichia canis; (c) catarata en paciente diabético con glaucoma secundario; (d) catarata secundaria a uveítis crónica con calcificación capsular

El trauma ocular, independiente de su presentación, puede predisponer a uveítis que genera inflamación en la cámara anterior, edema corneal, sinequias, y el correspondiente glaucoma asociado a aumento de la presión intraocular, hifema y desprendimiento de retina; adicionalmente, muchos pacientes desarrollan luxación de cristalino y desprendimiento de retina posterior a este evento (Slatter, 2008). De otra parte, la presencia de hemoparásitos genera una uveítis severa y trombocitopenia que causan hemorragias retinales (Ygreda, 2019), aumentando la presión intraocular y generando luxación de cristalino, adicional a los signos neurológicos asociados al parásito en contacto con médula espinal y encéfalo, que afectan directamente la visión del paciente y su estado de conciencia (Guerrero, 2016).

Según la literatura, las cataratas en estadios iniciales suelen presentarse en cápsula anterior o posterior o en ambas $\mathrm{y}$, a medida que se aumenta la degeneración cristalina, abarca lugares como el núcleo y la corteza (Donzel et al., 2017). En el presente estudio se tuvo $23.2 \%$ de cataratas ubicadas en el núcleo, hallazgo directamente relacionado con la edad avanzada de los pacientes. 
En el presente estudio, la mayoría de las cataratas fueron de tipo incipiente, donde la característica principal es que se observa una opacidad blanca focal (10 al 15\% de la superficie del cristalino) en el centro del globo (a la derecha del reflejo del flash). En este caso en particular, solo algunas fibras cristalinas están involucradas por lo que la visión no se encuentra afectada (Slatter, 2008). Las cataratas de tipo maduro, segundas en frecuencia, tiene como característica un lente totalmente opaco y sin reflejo tapetal, y como consecuencia la ceguera funcional, aunque es posible que se provoque una reacción pupilar a la luz (Gelatt, 2003).

Es importante resaltar a partir de estos hallazgos que, aunque la catarata de tipo incipiente fue la más reportada en el presente estudio, su diagnóstico es el más difícil de lograr, considerando que, al no generar cambios aparentes en el ojo ni de tipo comportamental en el perro, no es razón de consulta especializada por parte de los propietarios y se evidencia en hallazgos incidentales de pacientes que consultan por otras patologías oculares.

\section{Literatura Citada}

1. Abrams KL, Stabila PF, Kauper K, Elliott S. 2011. Vascular endothelial growth factor in diabetic and nondiabetic canine cataract patients. Vet Ophthalmol 14:93-99. doi: 10.1111/j.1463-5224.2010.00846.x

2. Acevedo SP, Ramírez, M, Restrepo LG 2009. Uveitis and glaucoma associated to Hepatozoon canis infection: a case report. Rev Colomb Cienc Pec 22: 287-295.

3. Bayón Del Río A, Palao C, Micó C, Vecino E. 2010. Oftalmología veterinaria: de la catarata al OCT. Arch Soc Esp Oftalmol 85: 387-389.

4. Davidson MG, Nelms SR. 2013. Diseases of the lens and cataract forma-tion. In: Gelatt KN, Gilger BC, Kern
TJ (eds). Veterinary ophthal-mology. $5^{\text {th }}$ ed. Ames: Wiley-Blackwell. p 1199-1233.

5. Del Sole M. 2014. Cataratas en perros. Universidad Nacional del centro de la Provincia de Buenos Aires. [Internet]. Disponible en: https://www.unicen.edu.ar/content/cataratas-en-perros

6. Donzel E, Arti L, Chahory S. 2017. Epidemiology and clinical presentation of canine cataracts in France: a retrospective study of 404 cases. Vet Ophthalmol 20: 131-139. doi: 10.1111/vop.12380

7. Espejo V, Esteban J. 2008. Diagnóstico precoz de las enfermedades oculares en pequeños animales. Colegio Oficial de Veterinarios de Madrid. [Internet]. Disponible en: http://www.colvema.org/ PDF/2026OJOS.pdf

8. Gelatt KN. 2003. Enfermedades y cirugía del cristalino en el perro. Fundamentos de oftalmología veterinaria. London: Manson Publishing. 227 p.

9. Gelatt KN, MacKay EO. 2005. Prevalence of primary breed related cataracts in the dog in North America. Vet Ophthalmol 8: 101-111. doi: 10.1111/ j.1463-5224.2005.00352.x

10. Gelatt KN, Gelatt J. 2011. Veterinary ophthalmic surgery. Philadelphia: Saunders. 476 p.

11. Glover TL, Davidson MG Nasisse MP, Olivero DK. 1995. La extracción intracapsular de lentes desplazadas en perros: un estudio retrospectivo de 57 casos (1984-1990). J Am Anim Hosp Assoc 31: 77-81. doi: 10.5326/15473317-31-1-77

12. Glover T, Constantinescu G. 1997. Surgery for cataracts. Vet Clin N AmSmall 27: 1143-1173. doi: 10.1016/S01955616(97)50107-0

13. Gómez LF, Atehortua CG, Padilla SC. 2007. La influencia de las mascotas en la vida humana. Rev Col Cienc Pec 20: 377-386.

14. Gomes MC, De Souza M, Vasconcelos RH, Bezerra WGA, Costa PPC. 2017. Aspectos e estágios da catarata em cãesRevisão de literatura. RBHSA 12: 456-471. 
15. Guerra R, Cabeças R, Diaz, J, Knott T, Freitas I, Vilhena H, Duarte $S$. 2018. Cataracts in Labrador Retriever and Jack Russell Terrier from the United Kingdom: a two-year retrospective study. Top Companion Anim M 133: 109-113. doi: $10.1053 /$ j.tcam.2018.09.001

16. Guerrero C. 2016. Problemática de la ehrlichiosis canina vista desde el aspecto teórico y el aspecto clínico en una clínica veterinaria de Bogotá. Tesis de Médico Veterinario. Bogotá: UDCA. 38 p.

17. Herrera D. 2007. Oftalmología clínica en animales de compañía. Buenos Aires: Intermédica. $276 \mathrm{p}$.

18. Komnenou AA, Mylonakis ME, Kouti $V$, Tendoma L, Leontides L, Skountzou E, Ofri R. 2007. Ocular manifestations of natural canine monocytic ehrlichiosis (Ehrlichia canis): a retrospective study of 90 cases. Vet Ophthalmol 10: 137142.doi: $10.1111 /$ j. 1463-5224.2007.00508.x

19. Lima-Gómez V, Ríos-González LC. 2004. Opacidad de cristalino en diabéticos. Prevalencia y asociación con deficiencia visual y retinopatía. Cir Ciruj 72: 171-175.
20. Mellersh CS. 2014. The genetics of eye disorders in the dog. Canine Genet Epidemiol 1: 3. doi: $10.1186 / 2052-6687-1-3$

21. Nelson JD. 2004. El ojo seco: ¿un dilema de diagnóstico o de definición? Arch Soc Esp Oftalmol 79: 589-590.

22. Raghuvanshi PDS, Maiti SK. 2013. Canine cataract and its management: an overview. J Anim Research 3: 17-26.

23. Slatter D. 2008. Slatter's fundamentals of veterinary ophthalmology. $6^{\mathrm{a}}$ ed. St. Louis, Missouri: Saunders. 584 p.

24. Silva TMF. 2010. Catarata em cães: revisão de literatura. PUBVET 4(2). 9 p. [Internet]. Disponible en: https:// www.pubvet.com.br/uploads/5f551865e91264d7ab655421cedea434.pdf

25. Ygreda GL. 2019. Uveítis en paciente canino con diagnóstico de Ehrlichia canis atendido en la Clínica Veterinaria. Tesis de Médico Veterinario. Lima: Univ. Peruana Cayetano Heredia. $15 \mathrm{p}$

26. Yoon S, Fleeman LM, Wilson BJ, Mansfield CS, McGreevy P. 2020. Epidemiological study of dogs with diabetes mellitus attending primary care veterinary clinics in Australia. Vet Rec 187: e22. doi: 10.1136/vr.105467 